\title{
Reserve-related activities and MRI metrics in multiple sclerosis patients and healthy controls: an observational study
}

\author{
Carolyn E. Schwartz ${ }^{1,2^{*}}$, Michael G. Dwyer ${ }^{3,4}$, Ralph Benedict ${ }^{5}$, Bianca Weinstock-Guttman ${ }^{5}$, Niels P. Bergsland ${ }^{3,6,7}$, \\ Jei Li ${ }^{1,8}$, Murali Ramanathan ${ }^{5,9}$ and Robert Zivadinov ${ }^{3,5,10}$
}

\begin{abstract}
Background: To examine whether past and current reserve-related activities make the brain less susceptible to MS pathology (i.e., lesions or disease-related atrophy).

Methods: This secondary analysis of a cohort study included 276 healthy controls (HC), and 65 clinically isolated syndrome (CIS), 352 relapsing-remitting MS (RR) and 109 secondary- progressive MS (SPMS) patients. Past reserverelated activities comprised educational and occupational attainment. Current reserve-related activities comprised strenuous and non-strenuous activities. MRI was performed on 3 T scanner. Regression and non-parametric analysis examined relationships between MRI metrics and reserve-related activities.

Results: Multivariate models ( $\mathrm{HC}$ as referent) revealed significant interactions in predicting strenuous reserve-related activities with chronic lesion burden (for CIS), brain- (for RR \& SPMS), subcortical- (for CIS, RR, \& SPMS) and amygdala(for RR) volumes. Maximal Lifetime Brain Growth was higher for RR patients who engaged in running before and after diagnosis, rather than only before or never. Residual Brain Volume was higher in RR patients who did weights-exercise before and after diagnosis, as compared to only before.

Conclusions: Reserve-related activities are related to brain health cross-sectionally in all MS subgroups, and longitudinally in RR patients. Consistent with reserve theory, RR patients who maintained strenuous activities had higher Maximal Lifetime Brain Growth and Residual Brain Volume. The study's limitations are discussed, including the potential for recall bias and design limitations that preclude causal inference.
\end{abstract}

\section{Background}

A growing body of research suggests that reserve is a key concept underlying resilience to neurological disease [1]. Conceptualized as a buffer between measurable disease pathology and actual level of function, reserve can be studied by investigating brain maintenance [2]. Maintenance theories emphasize neuro-protective mechanisms. Current conceptualizations of reserve posit that past enrichment activities and current stimulating leisure activities play an important role in helping an individual to build and maintain reserve [3]. These "reserve-related activities" in theory bring both history and current activities

\footnotetext{
* Correspondence: carolyn.schwartz@deltaquest.org

'DeltaQuest Foundation, Inc, 31 Mitchell Road, Concord, MA 01742, USA

${ }^{2}$ Departments of Medicine and Orthopaedic Surgery, Tufts University Medical

School, Boston, MA, USA

Full list of author information is available at the end of the article
}

into focus. In recent work, these activities are operationalized in terms of past activities (e.g., educational and occupational attainment) and current activities that are considered stimulating [4-7].

Recent research has documented a protective effect of stimulating leisure activities [6] in a broad range of disability domains in multiple sclerosis (MS) [4], such as physical, creative, intellectual, spiritual, and cultural enrichment. While both past and current reserve-related activities had notable cross-sectional and longitudinal associations with health and well-being, the current activities trumped past activities in explaining variance in health outcomes $[4,8]$. Reserve-related activities provide a fundamental resource to the individual [9], and are associated with better health habits, maintaining employment, and living independently [8]. Distinct from different aspects of insight into one's condition [10], high 
reserve-related activity engagement is associated with underlying cognitive appraisal processes that focus on the positive and more controllable aspects of their condition [11].

Longitudinal data supported a significant buffering effect of reserve, such that high-reserve individuals showed slower disability progression over six years of semi-annual follow-up [4]. These findings support the idea that symptom burden worsens as damage to the brain accumulates, and when reserve is exhausted, the progressive stage of the disease begins [12]. To date, however, very little research has addressed how these reserve-related activities relate to MRI metrics of brain health. Sumowski and colleagues reported that the negative effect of brain atrophy on information processing efficiency was attenuated at higher levels of reserve operationalized as education, such that MS subjects with higher levels of education were able to better withstand MS neuropathology without suffering cognitive impairment [13]. Further, early life enriching leisure activities were found to be more protective against memory deficits than cognitive inefficiency [14]. A retrospective analysis of neuroimaging data suggested that recreational activities protect against brain atrophy's detrimental influence on cognition [15].

All of these prior studies suffered from small samples sizes and limited operationalizations of reserve or reserverelated activities. We thus sought to use a large and rich data set to test the hypothesis that engaging in past or current reserve-related activities will make the brain less susceptible to MS pathology in the forms of lesions or disease-related atrophy. The study data set includes a large sample with MRI data, including a substantial reference group of healthy controls who provide important referent information for the multivariate analyses. It includes a novel measure estimating residual brain volume, and includes data on exercise that captured events and pattern changes before and after MS diagnosis. Building on an initial descriptive study of differences in reserve-related activities between healthy individuals and people with MS, the present work examines how these reserve-related activities relate to MRI metrics of Maximal Lifetime Brain Growth and Residual Brain Volume.

\section{Methods}

\section{Sample}

This secondary analysis utilized data from an ongoing prospective study of cardiovascular, environmental and genetic risk factors in MS at the MS Center of the State University of New York at Buffalo which enrolled over 1,000 subjects with clinically isolated syndrome (CIS) [16-18], MS, healthy controls, and other neurologic diseases $[19,20]$. For the purpose of this analysis, we focused on comparing people with CIS and MS to healthy controls. The sample included $65(8 \%)$ people with CIS; 352 (44\%) people with relapsing-remitting MS (RRMS); and 109 people (14 \%) with secondary progressive MS (SPMS). There were also 276 (34 \%) age- and gender-matched healthy controls. The inclusion criteria for this sub-analysis were presence of sufficient questionnaire data to obtain past and current reserve-related activity estimates. The exclusion criteria were presence of relapse and steroid treatment in the 30 days preceding study - entry- MRI for CIS and MS patients, pre-existing medical conditions known to be associated with brain pathology (cerebrovascular disease, positive history of alcohol abuse), and pregnancy. Healthy controls needed to meet the health-screen requirements, and had to have a normal physical and neurological examination. They were recruited from hospital personnel, or were respondents to a local advertisement.

\section{Procedure}

All subjects were assessed with a structured epidemiologic questionnaire administered in-person by a trained interviewer unaware of the subjects' disease status [16]. This procedure was aimed at minimizing missing data.

\section{Patient consents}

This study was approved by the local State University of New York Buffalo Health Sciences Institutional Review Board (HSIRB \#NEU2490109A) and written informed consent was obtained from all subjects.

\section{Measures}

Patient-reported In addition to demographic characteristics, the questionnaire queried physically strenuous (i.e., exercise) activities as well as strenuous and nonstrenuous activities (e.g., hobbies or other pastimes), similar to those included in questionnaires investigating reserve $[8,21]$. Based on the psychometric analyses described in [5], we created a derived measure of reserve-related activities comprised of three summary scores. These summary scores represented composite scores comprised of average scores for each factor score resulting from a principal components factor analysis. The first summary score was Past Reserve-related Activities, which summarized educational and occupational attainment. The second was Current Strenuous Reserve-related Activities, which included items for currently endorsed contact sports, aerobics, swimming, and wrestling. The third was Current Non-Strenuous Reserve-related Activities, which included items for currently endorsed reading, browsing the internet, meditation, and doing puzzles. These summary scores ranged from $2-10,0-5.4$, and $0-13.5$, respectively. These scores reflect frequency of endorsement on an ordinal scale. Table 1 shows the descriptive statistics by group. 
Table 1 Study participant demographics

\begin{tabular}{|c|c|c|c|c|c|c|c|}
\hline Variable & $\mathrm{HC}$ & $\mathrm{CIS}$ & RRMS & SPMS & Test Statistic & $P$-value & $P$-value ${ }^{a}$ \\
\hline N & 276 & 65 & 352 & 109 & & & \\
\hline Gender: \% female & 60.87 & 61.54 & 64.77 & 61.47 & 2.97 & 0.81 & \\
\hline Mean Age (sd) & $46.93(15.76)$ & $39.38(10.99)$ & $44.17(10.75)$ & $53.91(8.69)$ & 23.62 & 0.00 & $* * *$ \\
\hline Mean Age at Diagnosis (sd) & NA & $36.25(10.92)$ & $34.90(9.41)$ & $36.21(10.09)$ & 1.03 & 0.36 & \\
\hline Median EDSS (IQR) & NA & $1.50(1-2)$ & $2(3-1.5)$ & $6(5-6.5)$ & 24.64 & 0.00 & $* * *$ \\
\hline Diease Duration: mean no. years (sd) & NA & $5.65(3.32)$ & $13.1(7.11)$ & $21.85(9.10)$ & 83.61 & 0.00 & $* * *$ \\
\hline Range & NA & $3-20$ & $2-41$ & $5-45$ & & & \\
\hline Mean Body Mass Index (sd) & $27.31(5.70)$ & $27.14(5.86)$ & $27.40(5.93)$ & $26.10(6.01)$ & 1.35 & 0.26 & \\
\hline Employment Status \% & & & & & 201.79 & 0.00 & $* * *$ \\
\hline Full-time & 48.22 & 61.9 & 45.38 & 11.32 & & & \\
\hline Part-time & 15.81 & 11.11 & 12.14 & 10.38 & & & \\
\hline Homemaker & 3.16 & 7.94 & 3.47 & 4.72 & & & \\
\hline Student & 7.91 & 4.76 & 2.02 & 0 & & & \\
\hline Unemployed & 8.3 & 6.35 & 6.65 & 5.66 & & & \\
\hline Retired & 13.83 & 3.17 & 6.65 & 24.53 & & & \\
\hline Disabled & 0 & 3.17 & 21.97 & 41.51 & & & \\
\hline Other & 2.77 & 1.59 & 1.73 & 1.89 & & & \\
\hline Education \% & & & & & 30.85 & 0.01 & * \\
\hline High school not completed & 1.99 & 1.59 & 3.78 & 7.55 & & & \\
\hline Graduated high school & 15.54 & 12.7 & 17.15 & 21.7 & & & \\
\hline Some college/Associate/Technical Degree & 34.66 & 38.1 & 36.05 & 33.96 & & & \\
\hline Bachelor's degree & 25.9 & 30.16 & 23.26 & 19.81 & & & \\
\hline Graduate/Post-graduate & 21.91 & 17.46 & 19.77 & 16.98 & & & \\
\hline Race \% & & & & & 39.52 & 0.00 & *** \\
\hline Caucasian & 83.59 & 92.06 & 91.91 & 95.28 & & & \\
\hline Hispanic/Latino & 1.56 & 95.24 & 2.02 & 0.94 & & & \\
\hline Black/African-American & 8.59 & 98.41 & 4.91 & 2.83 & & & \\
\hline Asian & 3.91 & 100 & 0.58 & 0 & & & \\
\hline American Indian/Alaska Native & 0.39 & 0 & 0 & 0 & & & \\
\hline Other & 1.95 & 0 & 0.58 & 0.94 & & & \\
\hline Past reserve-related Activities: Mean (sd) & $6.60(0.13)$ & $6.44(1.76)$ & $6.27(2.06)$ & $5.75(2.04)$ & 4.39 & 0.00 & $* * *$ \\
\hline Range & $2-10$ & $3-10$ & $2-10$ & $2-10$ & & & \\
\hline Strenuous reserve-related Activities: Mean (sd) & $1.61(0.078)$ & $0.70(0.67)$ & $0.64(0.70)$ & $0.36(0.41)$ & 76.73 & 0.00 & *** \\
\hline Range & $0-5.42$ & $0-2.83$ & $0-3.23$ & $0-1.8$ & & & \\
\hline Non-Strenuous reserve-related Activities: Mean(sd) & $5.80(0.16)$ & $5.18(2.47)$ & $5.36(2.62)$ & $5.53(2.84)$ & 1.64 & 0.18 & \\
\hline Range & $0.5-12.67$ & $0-12$ & $0-13.5$ & $0-12.83$ & & & \\
\hline
\end{tabular}

aHC Healthy Control, CIS Clinically Isolated Syndrome, RRMS Relapsing Remitting MS, SPMS Secondary Progressive MS, The F-Statistics and P-values shown are from tests for differences between disease group for the given variable

${ }^{*} p<0.05$; ** $p<0.01$; *** $p<0.001$

Neuroimaging Full details of the scan protocol have been previously reported [22]. Briefly, fluid-attenuated inversion recovery, T1 spin echo (pre and post gadolinium injection) and 3D-T1 images were acquired on a 3 T GE Signa Excite HD 12.0 Twin Speed 8-channel scanner (General Electric, Milwaukee, WI, USA). T2, T1, and gadolinium-enhancing-lesion volumes (LV) were calculated using a reliable semi-automated edge detection contouring/thresholding technique using JIM software. We used SIENAX to obtain normalized tissue volumes of the brain, gray and white matter as well as the lateral ventricles. The 3D-T1 images were preprocessed using a 
lesion filling technique to reduce the impact of white matter lesions on volumetric segmentations [23]. Tissue volumes for the bilateral thalamus, caudate, putamen, globus pallidus, hippocampus, amygdala and nucleus accumbens were calculated using FMRIB's FIRST, a model-based segmentation and registration tool [24].

\section{Statistical analysis}

Descriptive analyses Descriptive statistics of demographic variables were computed by disease group, and linear models were used to compare group values for each demographic variable.

\section{Maximal lifetime brain growth}

As previously reported [25], and consistent with other researchers [14], we used the SIENAX scaling factor as a surrogate for intracranial volume (ICV) to determine Maximal Lifetime Brain Growth, since ICV is a reliable estimate of Maximal Lifetime Brain Growth. This metric reflects the maximum brain volume attained in the individual's lifetime, prior to brain atrophy associated with aging and disease progression. In terms of reserve theory [3], this metric would be considered brain reserve. This variable was transformed into gender-specific z-scores in order to adjust for the expected difference in ICV [25].

\section{Residual brain volume}

We used a regression technique to produce a measure of residual brain volume [26]. Briefly, we regressed normalized brain parenchymal volume (NBPV) against T2 lesion volume, age, EDSS, MS disease duration, and gender to produce per-subject measures of expected brain volume based on demographic and overall MS disease severity. Then, we subtracted this expected NBPV from the observed NBPV. Thus, negative values represent more brain atrophy than expected, and positive values represent less. In terms of reserve theory [3], this metric would be related to modifiable reserve.

\section{Regression analyses}

Data reduction See supplementary text for full description of data reduction analyses done prior to inferential statistical analysis. These analyses yielded the following MRI variables for analysis: Chronic Brain Burden (T1 and T2 lesion volume), Active Brain Burden (Contrast-enhanced lesion volume), Brain Volume, and Subcortical Gray Matter Volume. They yielded past, current strenuous, and current non-strenuous reserve-building activities.

Multivariable analysis of MRI structural metrics To examine the relationship between MRI metrics, diseasegroup, and reserve-related activities, we computed regression models for each of the three reserve-related activities summary scores: past, current strenuous and current nonstrenuous. The model included relevant covariates (age and gender); MRI composite(s) (chronic lesion burden, active lesion burden, brain volume, and subcortical gray matter volume in separate models); dummy variables for MS disease group (CIS, RRMS, and SPMS), with healthy controls as the comparison/referent group; and finally the variables of particular interest in these analyses: the interaction terms between MS dummy variables and MRI metrics. These interaction terms test if there is a differential relationship between MRI and reserve metrics by MS disease group. The Type I error rate for each model was $5 \%$.

Investigating change in strenuous activities and brain structure in relapsing-remitting group We then created a variable that summarized change in strenuous activities from before and after MS diagnosis in relapsingremitting patients. Separate change variables were created for each of the strenuous activities (aerobics, running, walking, weights, yoga, and taichi); and medians were plotted by group for the above-described two derived MRI metrics of specific relevance to reserve: Maximal Lifetime Brain Growth and Residual Brain Volume.

The distributions of strenuous activities by change group were visualized using box plots, and then non-parametric tests were used to compare the distributions, first in an omnibus Kruskal-Wallis test and then in pairwise Wilcoxon tests [27]. This set of analyses was only implemented in the RR subgroup of MS patients for several reasons. First, this was the largest subgroup of MS patients in the sample $(n=352)$, and thus the analyses would have better statistical power to detect relevant differences. Second, reserve theory would predict that reserve is more intact in RR patients [12] and thus the hypothesized relationships between reserve-related activities, Maximal Lifetime Brain Growth, and current reserve would be clinically relevant.

All analyses were implemented using Stata 13 [28] and R Studio ${ }^{\text {th }}$ version 0.98.1103 [29].

\section{Results}

\section{Sample}

Table 1 provides the descriptive statistics on the study sample, as well as comparisons among groups. The sample included 276 healthy controls, 65 people with CIS, 352 with RR, and 109 with SPMS. The majority of the sample was female, and the mean age in the various subsamples ranged from 39-54 years. Mean age at diagnosis in the various subsamples ranged from 35 to 36 years, and median Expanded Disability Status Scale (EDSS) score in the various subsamples ranged from 1.5 to 6.0. The mean disease duration ranged from 5.7 to 21.9 years (range 2-45 years). The sample had a body- mass- index 
consistent with being overweight. The majority of the healthy controls, CIS and RR samples were employed either full- or part-time, but the employed SPMS cohort constituted only $22 \%$ of the sample. The majority of the sample had some college education or greater, and the sample was predominantly Caucasian. The subsamples included in this study differed by age, EDSS, disease duration, employment status, education, and race.

\section{Reserve metrics descriptive statistics and correlations by group}

Mean scores and score ranges for the various reserverelated activities are shown in Table 1 . The lowest score ranges were in the current strenuous reserve-related activities (range 0-5.4), and healthy controls reported the highest scores in these activities, followed by CIS, RR, and SPMS patients, respectively $(p<0.001)$. There were no differences between subsamples in engagement in non-strenuous activities, but group differences were found in past-reserve building activities, with SPMS patients reported the lowest scores $(p<0.01)$.

\section{Multivariable analysis of MRI structural metrics}

Mean scores and standard deviations for the various MRI metrics are provided in Additional file 1: Table S1. Results of the regression models suggest that there are no significant predictors of past reserve-related activities or non-strenuous reserve-related activities (Table $2 \mathrm{a}$ and c). Consequently, the regression models explained almost no variance in past and non-strenuous reserve-related activities. In contrast, the models predicting strenuous reserverelated activities suggested that there was a differential positive association for CIS with chronic lesion burden; a differential negative association for RR and SPMS with brain volume; and a differential negative association with CIS, RR, and SPMS with subcortical gray matter volume, and with $\mathrm{RR}$ and amygdala volume (Table 2b). Further, the models explained $10 \%$, $9 \%$, $28 \%$, and $29 \%$ of the variance in strenuous reserve-related activities, after adjusting for the number of predictors (Table 2a-c). There were no significant group-by-active lesion interactions predicting strenuous reserve-related activities.

\section{Relapsing-remitting group-specific analyses of MRI reserve metrics}

Figures 1a-e show box plots of Maximal Lifetime Brain Growth (maximum lifetime brain growth) by change in strenuous activity group for aerobics, running, walking, weights, and yoga. On the far left is the box plot for individuals who engaged in the strenuous activity both before and after MS diagnosis. The box plot immediately to the right is for those individuals who engaged only after, then only before, then never. The far right box plot show the distribution on the Maximal Lifetime Brain Growth score for those individuals missing data on that activity.

There were significant differences between groups on aerobics (Kruskal Wallis $\chi^{2}=14.57, p=0.01$ ), such that the Never group differed from the Only-After and OnlyBefore groups and the Missing group differed from all but the Never groups (Additional file 2: Table S2). There were significant differences between groups on running (Kruskal Wallis $\chi^{2}=11.77, p=0.02$ ), such that the Before-and-After Group differed from the Only-Before and Never groups, and the Missing group differed from the Only-Before and Never groups (Additional file 2: Table S2). The non-parametric comparisons for yoga showed trend significance (Kruskal Wallis $X^{2}=8.69$, $p=0.07)$, with the differences being between Beforeand-After and Only-after versus Missing. There were no significant differences in the Walking or Weights change groups with respect to Maximal Lifetime Brain Growth.

Figures 2a-e show box plots of the Residual Brain Volume by change in strenuous- activity group for aerobics, running, walking, weights, and yoga, set up identically to Fig. 1a-e. There was a significant difference between groups on weights (Kruskal Wallis $\chi^{2}=10.8, p=0.03$ ), such that the Only-Before group differed from the Before-and-After and the Never groups (Additional file 2: Table S2). There were no significant differences in any of the other strenuous change groups and Residual Brain Volume.

\section{Discussion}

Our findings suggest that MRI metrics related to chronic lesion burden, brain volume, and subcortical gray matter volume were differentially associated with MS groups in predicting strenuous reserve-related activities, but not for past activities or for current non-strenuous activities. A closer look at changes in strenuous activities over time in RR patients suggests that among those individuals who provided data on these variables, people who engaged in running before and after diagnosis, rather than only before or never, had higher Maximal Lifetime Brain Growth. That is, their current activity is associated with pre-morbid brain size. People who engaged in aerobics only after or only before differed from those who never engaged in aerobics. Among those individuals who provided data on these variables, individuals who engaged in weights-related exercise before and after diagnosis, as compared to only before or never had lower Residual Brain Volume. These findings suggest that reserve-related activities are related to brain health both cross-sectionally in MS subgroups, and with regard to changes in activities over time in RR patients. These findings support the importance of environmental factors in facilitating reserverelated activities and potentially buffering individuals from disease burden and progression. 
Table 2 a-c Regression models predicting reserve-related activities ${ }^{2}$

\begin{tabular}{|c|c|c|c|c|c|c|c|c|c|c|c|}
\hline \multicolumn{12}{|l|}{ Dependent Variable: } \\
\hline \multicolumn{4}{|c|}{ 2a Past Reserve Building Activities } & \multicolumn{4}{|c|}{ 2b Strenuous Current reserve-related Activities } & \multicolumn{4}{|c|}{ 2c Non-Strenuous Current reserve-related Activities } \\
\hline Predictors & $\beta$ & $\mathrm{t}$ & $p$ & Predictors & $\beta$ & $\mathrm{t}$ & p & Predictors & $\beta$ & $\mathrm{t}$ & $p$ \\
\hline \multicolumn{12}{|c|}{ Chronic lesion burden models } \\
\hline Age & 0.01 & 0.13 & 0.90 & Age & -0.19 & -3.94 & 0.00 & Age & -0.05 & -0.94 & 0.35 \\
\hline Gender & -0.02 & -0.42 & 0.68 & Gender & -0.07 & -1.72 & 0.09 & Gender & -0.05 & -1.07 & 0.29 \\
\hline Chronic Burden & -0.31 & -1.25 & 0.21 & Chronic Burden & -0.34 & -1.46 & 0.15 & Chronic Burden & 0.20 & 0.82 & 0.41 \\
\hline $\mathrm{CIS}$ & 0.01 & 0.08 & 0.94 & $\mathrm{CIS}$ & -0.37 & -5.04 & 0.00 & $\mathrm{CIS}$ & -0.03 & -0.43 & 0.67 \\
\hline RR & -0.03 & -0.24 & 0.81 & $\mathrm{RR}$ & -0.51 & -5.33 & 0.00 & RR & 0.09 & 0.87 & 0.38 \\
\hline PMS & -0.14 & -1.37 & 0.17 & PMS & -0.51 & -5.42 & 0.00 & PMS & 0.13 & 1.32 & 0.19 \\
\hline Chronic X CIS & 0.01 & 0.18 & 0.86 & Chronic X CIS & 0.15 & 2.58 & 0.01 & Chronic X CIS & 0.06 & 1.05 & 0.29 \\
\hline Chronic X RR & 0.17 & 0.85 & 0.39 & Chronic X RR & 0.26 & 1.41 & 0.16 & Chronic X RR & -0.14 & -0.70 & 0.49 \\
\hline Chronic X SPMS & 0.30 & 1.42 & 0.16 & Chronic X SPMS & 0.30 & 1.51 & 0.13 & Chronic X SPMS & -0.17 & -0.83 & 0.41 \\
\hline Adjusted R-squared & 0.00 & & & Adjusted R-squared & 0.10 & & & Adjusted R-squared & 0.00 & & \\
\hline $\mathrm{N}$ & 523 & & & $\mathrm{~N}$ & 526 & & & $\mathrm{~N}$ & 535 & & \\
\hline \multicolumn{12}{|c|}{ Active lesion burden models } \\
\hline Age & 0.00 & -0.09 & 0.93 & Age & -0.18 & -3.83 & 0.00 & Age & -0.07 & -1.40 & 0.16 \\
\hline Gender & -0.01 & -0.33 & 0.74 & Gender & -0.08 & -1.84 & 0.07 & Gender & -0.04 & -0.88 & 0.38 \\
\hline Active Burden & -0.04 & 0.00 & 1.00 & Active Burden & -11.72 & -0.89 & 0.38 & Active Burden & -4.71 & -0.34 & 0.73 \\
\hline $\mathrm{CIS}$ & -0.09 & -0.81 & 0.42 & $\mathrm{ClS}$ & -0.48 & -4.95 & 0.00 & $\mathrm{CIS}$ & -0.10 & -0.99 & 0.32 \\
\hline $\mathrm{RR}$ & -0.17 & -1.09 & 0.28 & RR & -0.75 & -5.39 & 0.00 & RR & -0.10 & -0.67 & 0.50 \\
\hline PMS & -0.22 & -1.55 & 0.12 & PMS & -0.73 & -5.59 & 0.00 & PMS & -0.03 & -0.25 & 0.81 \\
\hline Active $X$ CIS & 0.01 & 0.00 & 1.00 & Active $X \mathrm{ClS}$ & 2.81 & 0.9 & 0.37 & Active $\times$ CIS & 1.02 & 0.34 & 0.73 \\
\hline Active $X$ RR & 0.01 & 0.00 & 1.00 & Active $X \mathrm{RR}$ & 11.42 & 0.89 & 0.38 & Active $X$ RR & 4.56 & 0.34 & 0.73 \\
\hline Active $X$ SPMS & -0.02 & -0.03 & 0.97 & Active $X$ SPMS & 0.51 & 0.84 & 0.40 & Active $X$ SPMS & 0.15 & 0.24 & 0.81 \\
\hline Adjusted R-squared & -0.01 & & & Adjusted R-squared & 0.09 & & & Adjusted R-squared & 0.00 & & \\
\hline N & 504 & & & $\mathrm{~N}$ & 505 & & & $\mathrm{~N}$ & 514 & & \\
\hline \multicolumn{12}{|l|}{ Brain volume models } \\
\hline Age & 0.03 & 0.66 & 0.51 & Age & -0.13 & -3.12 & 0.00 & Age & 0.03 & 0.55 & 0.58 \\
\hline Gender & -0.02 & -0.52 & 0.60 & Gender & 0.01 & 0.45 & 0.65 & Gender & -0.01 & -0.32 & 0.75 \\
\hline Brain Volume* & 0.09 & 1.00 & 0.32 & Brain Volume* & 0.22 & 3.08 & 0.00 & Brain Volume* & 0.10 & 1.16 & 0.25 \\
\hline White Matter Vol. & -0.01 & -0.09 & 0.93 & White Matter Vol. & 0.11 & 1.40 & 0.16 & White Matter Vol. & -0.10 & -1.02 & 0.31 \\
\hline $\mathrm{CIS}$ & 0.19 & 0.22 & 0.82 & $\mathrm{CIS}$ & 0.92 & 1.26 & 0.21 & $\mathrm{CIS}$ & 0.32 & 0.39 & 0.70 \\
\hline $\mathrm{RR}$ & -0.86 & -1.13 & 0.26 & $\mathrm{RR}$ & 0.98 & 1.59 & 0.11 & $\mathrm{RR}$ & -0.45 & -0.60 & 0.55 \\
\hline SPMS & 0.72 & 0.97 & 0.33 & SPMS & 1.20 & 1.98 & 0.05 & SPMS & -1.09 & -1.50 & 0.13 \\
\hline Brain Vol X CIS & -0.31 & -0.57 & 0.57 & Brain Vol X CIS & -0.61 & -1.34 & 0.18 & Brain Vol X CIS & -0.12 & -0.23 & 0.82 \\
\hline Brain Vol X RR & -0.04 & -0.09 & 0.93 & Brain Vol X RR & -1.01 & -2.58 & 0.01 & Brain Vol X RR & 0.06 & 0.13 & 0.90 \\
\hline Brain Vol X SPMS & -0.71 & -1.47 & 0.14 & Brain Vol X SPMS & -0.78 & -1.98 & 0.05 & Brain Vol X SPMS & -0.18 & -0.38 & 0.70 \\
\hline WMV X CIS & 0.11 & 0.16 & 0.87 & WMV X CIS & -0.60 & -0.97 & 0.33 & WMV X CIS & -0.25 & -0.40 & 0.69 \\
\hline WMV X RR & 0.86 & 1.25 & 0.21 & WMV X RR & -0.44 & -0.78 & 0.44 & WMV X RR & 0.35 & 0.52 & 0.61 \\
\hline WMV X SPMS & -0.12 & -0.20 & 0.84 & WMV X SPMS & -0.77 & -1.60 & 0.11 & WMV X SPMS & 1.25 & 2.19 & 0.03 \\
\hline Adjusted R-squared & 0.01 & & & Adjusted R-squared & 0.28 & & & Adjusted R-squared & 0.00 & & \\
\hline$N$ & 732 & & & N & 759 & & & $\mathrm{~N}$ & 757 & & \\
\hline
\end{tabular}


Table 2 a-c Regression models predicting reserve-related activities ${ }^{a}$ (Continued)

\begin{tabular}{|c|c|c|c|c|c|c|c|c|c|c|c|}
\hline \multicolumn{12}{|c|}{ Subcortical gray matter volume models } \\
\hline Age & 0.03 & 0.74 & 0.46 & Age & -0.15 & -4.11 & 0.00 & Age & -0.01 & -0.19 & 0.85 \\
\hline Gender & -0.02 & -0.43 & 0.67 & Gender & 0.02 & 0.70 & 0.49 & Gender & -0.01 & -0.38 & 0.71 \\
\hline Subcortical Health* & 0.12 & 1.10 & 0.27 & Subcortical Health* & 0.35 & 4.01 & 0.00 & Subcortical Health* & 0.01 & 0.11 & 0.91 \\
\hline Amygdala Vol. & -0.02 & -0.27 & 0.78 & Amygdala Vol. & -0.16 & -2.39 & 0.02 & Amygdala Vol. & 0.06 & 0.81 & 0.42 \\
\hline $\mathrm{CIS}$ & 0.00 & -0.01 & 1.00 & $\mathrm{ClS}$ & 0.60 & 1.26 & 0.21 & $\mathrm{CIS}$ & -0.27 & -0.45 & 0.65 \\
\hline RR & -0.27 & -0.47 & 0.64 & $\mathrm{RR}$ & 0.37 & 0.81 & 0.42 & RR & -0.03 & -0.05 & 0.96 \\
\hline SPMS & 0.37 & 0.72 & 0.47 & SPMS & 0.48 & 1.18 & 0.24 & SPMS & 0.05 & 0.11 & 0.91 \\
\hline Subcortical X CIS & 0.04 & 0.06 & 0.95 & Subcortical X CIS & -1.03 & -2.07 & 0.04 & Subcortical X CIS & 0.22 & 0.37 & 0.71 \\
\hline Subcortical X RR & 0.16 & 0.27 & 0.79 & Subcortical X RR & -1.57 & -3.43 & 0.00 & Subcortical X RR & 0.24 & 0.44 & 0.66 \\
\hline Subcortical X SPMS & -0.64 & -1.26 & 0.21 & Subcortical X SPMS & -1.29 & -3.12 & 0.00 & Subcortical X SPMS & -0.03 & -0.06 & 0.95 \\
\hline Amygdala X CIS & -0.06 & -0.17 & 0.87 & Amygdala X CIS & 0.15 & 0.52 & 0.61 & Amygdala X CIS & -0.01 & -0.04 & 0.97 \\
\hline Amygdala X RR & 0.08 & 0.22 & 0.83 & Amygdala X RR & 0.77 & 2.70 & 0.01 & Amygdala X RR & -0.27 & -0.79 & 0.43 \\
\hline Amygdala X SPMS & 0.19 & 0.58 & 0.56 & Amygdala X SPMS & 0.48 & 1.83 & 0.07 & Amygdala X SPMS & -0.04 & -0.12 & 0.90 \\
\hline Adjusted R-squared & 0.01 & & & Adjusted R-squared & 0.29 & & & Adjusted R-squared & -0.01 & & \\
\hline$N$ & 731 & & & N & 758 & & & $\mathrm{~N}$ & 756 & & \\
\hline
\end{tabular}

CIS, Clinically Isolated Syndrome, RRMS Relapsing Remitting MS, SPMS Secondary Progressive MS

anteraction terms are denoted by " $X$ ' between main effect terms in the bottom section of each table. The referent group is healthy controls

There are limitations to our findings which must be considered. First, this is an observational study that investigates a research question that has not been addressed before. Accordingly, we did not have a priori hypotheses, nor did we know how many comparisons we would make a priori; we thus did not adjust for multiple comparisons in our statistical analyses. We are simply reporting on what was observed in this relatively large observational cohort study. Future research by others might examine this research question in an independent sample and evaluate whether the effects detected are small, medium, or large effect sizes. Further, because the data are cross-sectional, they may suffer from biases associated with retrospective self-report. Our ability to make causal inferences is thus hindered: the findings could either reflect a buffering effect or reverse causality-that people with worse health outcomes participate in fewer stimulating leisure activities because of their worse health. Further, the confidence intervals for the 'never' group in all of the plots shown in Figs. 1a-e and 2a-e are very wide, suggesting that this is a 'noisy' estimate which should be interpreted with caution. Additionally, we did not assess neurocognitive status or other factors associated with brain health, such as genetic contributions or trauma. In the subgroup analysis focused on RR patients, our findings were consistent with our hypothesis, suggesting that higher Maximal Lifetime Brain Growth and current reserve are associated with maintaining strenuous activities in spite of having an MS diagnosis. It is equally possible, however, that people with more aggressive MS are less likely to continue strenuous activity because of physical disability or transient heat-related symptom worsening. Additionally, the varying sample sizes by MS group and missing data result in varying statistical power for the multivariate comparisons, and possible biases in the strenuous-change analyses. We tried to counteract this challenge by using non-parametric analyses, and by explicitly comparing results on the missingdata group.

It is notable that a large number of statistical comparisons were done, while relatively few statistically significant associations were found. While this study was initiated without a priori hypotheses that would have driven specific hypothesis- testing or power calculations aimed at detecting pre-specified effect sizes, the number of comparisons done might have led to false positive findings. Rather than employing one or another conservative adjustment for the Type I error rate, we simply describe our findings. We hope that the high level of transparency we have strived for regarding the analyses done will facilitate future research aimed at replicating and confirming the findings It is also possible that the MRI metrics used here are not sensitive enough to measure plasticity. Research on brain plasticity suggests that stimulating activities are associated with structural brain changes [30]. Strenuous activities, such as aerobic exercise, have been associated with increased neurogenesis in the dentate gyrus of the hippocampus, and upregulation of brain-derived neurotrophic factor [31]. Cognitive training has been found to result in focal volume changes in areas relevant to task demand [32, 33]. Barulli and Stern [2] suggest that these findings 


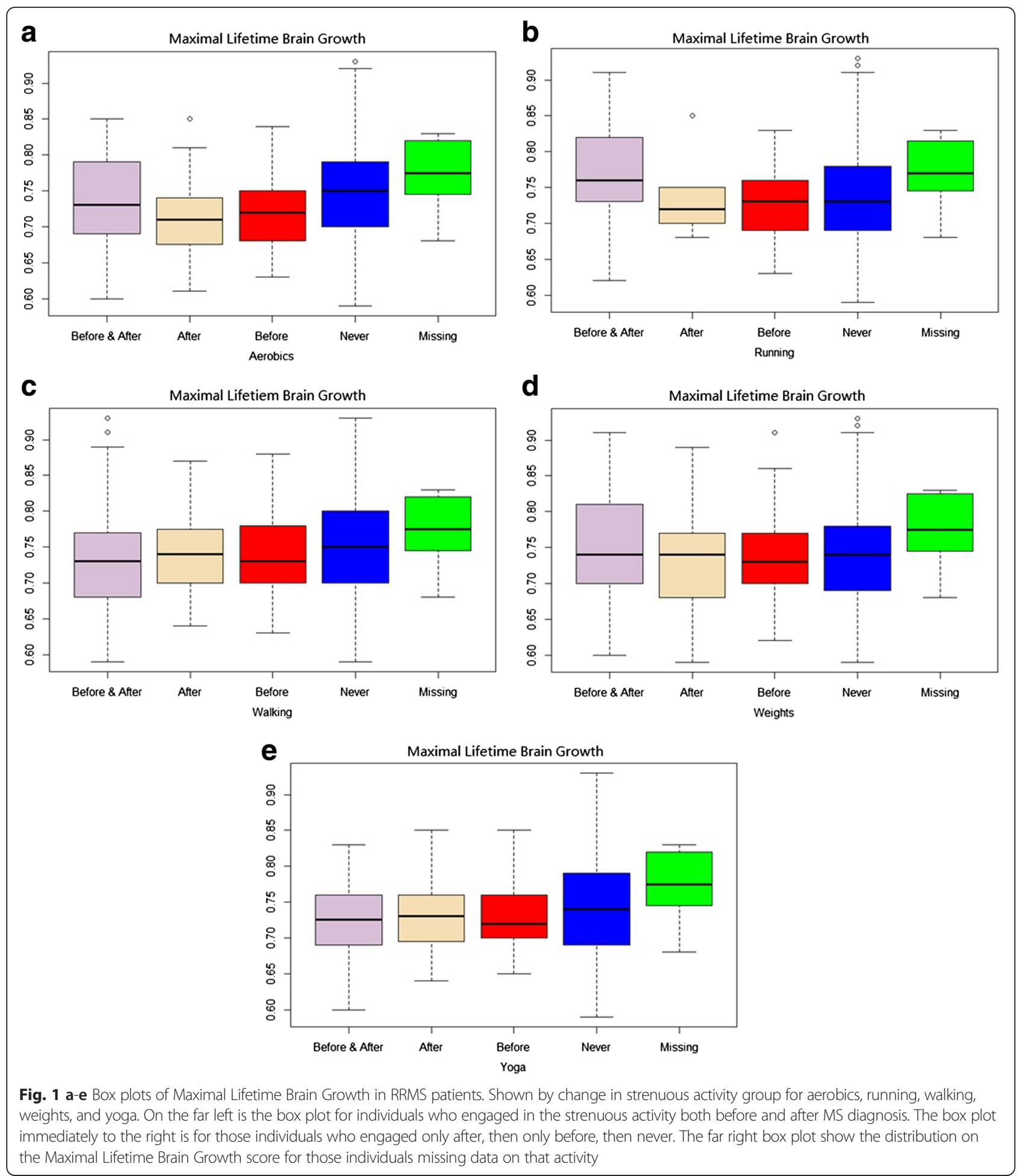

support the long-term benefits of intellectual stimulation and physical exercise, and that such exposures may not only help the brain to adapt to structural changes, but also may help to prevent those changes to begin with. Future research might use connectome structural and functional MRI measures to address reserve.
Despite the above limitations, the study's strengths are substantial: it has a large sample with MRI data, includes healthy controls as a reference group, utilizes novel MRI metrics, and captures exercise patterns before and after MS diagnosis to allow an evaluation of the its impact on brain health. 


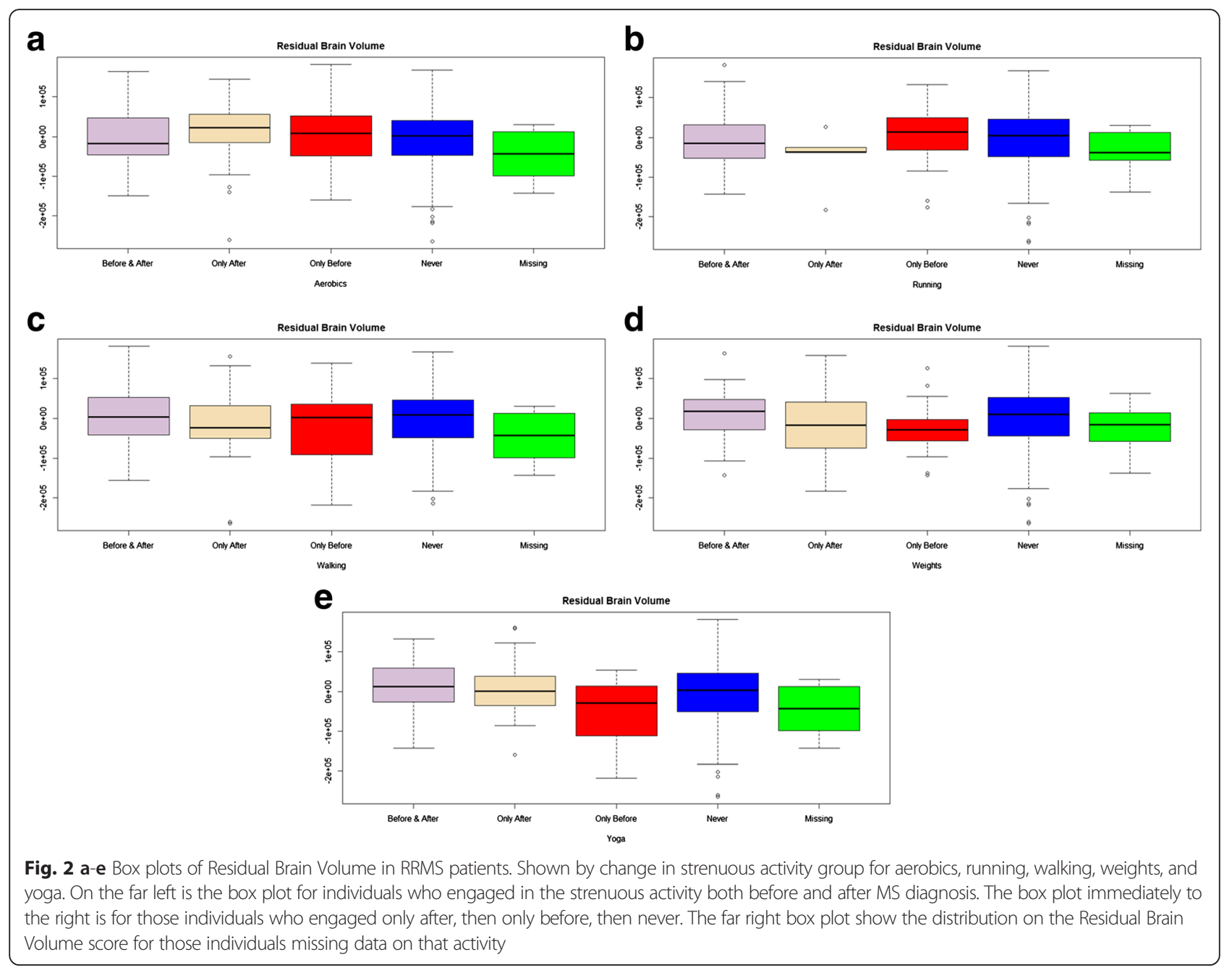

\section{Conclusions}

Our findings are suggestive that MRI metrics relate to reserve-related activities, although our cross-sectional design renders our findings somewhat unclear with regard to direction of association in the correlations or multivariate models. Prospective research is needed to definitively support the buffering effect of reserve-related activities, but we believe that the findings of the present study build on a growing evidence base that should encourage people with MS to maintain their engagement in a range of strenuous stimulating activities to promote health and prevent disability progression.

\section{Additional files}

Additional file 1: Table S1. Descriptive statistics of MRI measures. (XLS $10 \mathrm{~kb}$ )

Additional file 2: Table S2. Results of non-parametric tests comparing strenuous change groups in relapsing-remitting patients. (XLS $13 \mathrm{~kb}$ )

\section{Abbreviations}

CIS, clinically isolated syndrome; EDSS, expanded disability status scale; ICV, intracranial volume; MRI, magnetic resonance imagine; MS, multiple sclerosis; $\mathrm{RR}$, relapsing-remitting; SPMS, secondary progressive multiple sclerosis

\section{Acknowledgements}

Not applicable.

Funding

This study was sponsored by the Buffalo Neuroimaging Analysis Center research fund.

\section{Availability of data and materials}

Supporting documentation for our findings is provided in manuscript data and supplementary tables. Scientists wishing to gain access to our data may contact the senior author (RZ), who will consider such requests on a case-by-case basis, subject to the request's compliance with institutional requirements for participant confidentiality and scientific rigor of the proposed research question.

\section{Authors' contributions}

Study concept and design: CES, MGD, RB, BWG, NPB, MR, RZ. Acquisition of data: MGD, RB, BWG, NPB, MR, RZ. Analysis and interpretation: CES, MGD, RB, $B W G, N P B, J L, M R, R Z$. Critical revision of the manuscript for important intellectual 
content: CES, MGD, RB, BWG, NPB, JL, MR, RZ. Study supervision: CES, MGD, RZ. Statistical analyses completed by CS and JL, DeltaQuest Foundation. All authors read and approved the final manuscript.

\section{Competing interests}

The authors declare they have no competing interests and no financial competing interests.

Dr. Schwartz served on the scientific advisory board and serves on the speakers' bureau for EMD Serono; and has served as a consultant for Biogen; has received grant support from the National Multiple Sclerosis Society, Sunnybrook Health Sciences Centre, the Patient-Centered Outcomes Research Institute, the National Cancer Institute, the Accelerated Cure Project, and Biogen. She serves as Co-Editor-in-Chief of Quality of Life Research.

Dr. Dwyer has received personal compensation from Claret Medical and EMD Serono, and research grant support from Novartis.

Dr. Benedict Has received research support from the National Multiple Sclerosis Society, MS Federation, Accorda, Mallinckrodt, Novartis, Biogen, and Genzyme; has been a speaker for EMD Serono; has served as a consultant for Genentech Teva, Novartis, Biogen, and Genzyme; and has received royalties from Psychological Assessment Resources, Inc.

Dr. Weinstock-Guttman has participated in speaker's bureaus and served as a consultant for Biogen Idec, Teva Neuroscience, EMD Serono, Novartis, Genzyme \& Sanofi, Acorda Therapeutics, Inc. and Genentech. Dr. WeinstockGuttman also has received grant/research support from the agencies listed in the previous sentence as well as Questcor Pharmaceuticals, Inc., and Shire. She serves on the editorial board for BMJ Neurology, Journal of International MS and CNS Drugs.

Mr. Bergsland reports no disclosures.

Ms. Li reports no disclosures.

Dr. Ramanathan received research funding the National Multiple Sclerosis Society and the Department of Defense. He received compensation for serving as an Editor from the American Association of Pharmaceutical Scientists.

Dr. Zivadinov received personal compensation from Biogen Idec, Teva Pharmaceuticals, EMD Serono, Novartis, Claret Medical and Genzyme for speaking and consultant fees. Dr. Zivadinov received financial support for research activities from Biogen Idec, Teva Pharmacuticals, EMD Serono, Novartis, Claret Medical and Genzyme. Dr. Zivadinov serves on editorial board of J Alzh Dis, BMC Med, BMC Neurol, Vein and Lymphatics and Clinical CNS Drugs. He is Executive Director and Treasurer of International Society for Neurovascular Disease.

\section{Consent for publication}

Not applicable.

\section{Ethics approval and consent to participate}

This study was approved by the local State University of New York Buffalo Health Sciences Institutional Review Board (HSIRB \#NEU2490109A), and written informed consent was obtained from all subjects.

\section{Search terms}

Multiple sclerosis (41), case control studies (53), MRI (120), outcome research (112).

\section{Author details}

'DeltaQuest Foundation, Inc, 31 Mitchell Road, Concord, MA 01742, USA. ${ }^{2}$ Departments of Medicine and Orthopaedic Surgery, Tufts University Medical School, Boston, MA, USA. ${ }^{3}$ Buffalo Neuroimaging Analysis Center, Department of Neurology, School of Medicine and Biomedical Sciences, State University of New York, Buffalo, NY, USA. ${ }^{4}$ Department of Biomedical Informatics, University of Buffalo, State University of New York, Buffalo, NY, USA. ${ }^{5}$ Department of Neurology, School of Medicine and Biomedical Sciences, University of Buffalo, State University of New York, Buffalo, NY, USA. "Istituto Di Ricovero e Cura a Carattere Scientifico, "S.Maria Nascente", Don Gnocchi Foundation, Milan, Italy. ${ }^{7}$ Department of Electronics, Information and Bioengineering, Politecnico di Milano, Milan, Italy. ${ }^{8}$ Department of Biostatistics, University of Massachusetts, Amherst, MA, USA. ${ }^{9}$ Department of Pharmaceutical Sciences, School of Medicine and Biomedical Sciences, State University of New York, Buffalo, NY, USA. ${ }^{10}$ MR Imaging Clinical Translational Research Center, School of Medicine and Biomedical Sciences, University at Buffalo, State University of New York, Buffalo, NY, USA.
Received: 7 April 2016 Accepted: 24 June 2016

Published online: 19 July 2016

\section{References}

1. Stern Y. Cognitive reserve: Theory and applications. New York: Taylor \& Francis; 2007.

2. Barulli D, Stern Y. Efficiency capacity, compensation, maintenance, plasticity: emerging concepts in cognitive reserve. Trends Cogn Sci. 2013;17(10):502-09.

3. Schwartz CE, Rapkin BD, Healy BC. Reserve and Reserve-Building Activities Research: Key challenges and future directions. Under review. 2015

4. Schwartz CE, Quaranto BR, Healy BC, Benedict RH, Vollmer TL. Cognitive reserve and symptom experience in multiple sclerosis: a buffer to disability progression over time? Arch Phys Med Rehabil. 2013;94(10):1971-81.

5. Schwartz CE, Ayandeh A, Ramanathan M, Benedict RH, Dwyer MG, Weinstock-Guttman B, et al. Reserve-building activities in multiple sclerosis patients and healthy controls: A descriptive study. BMC Neurol. 2015;15:135.

6. Sumowski JF, Wylie GR, DeLuca J, Chiaravalloti N. Intellectual enrichment is linked to cerebral efficiency in multiple sclerosis: functional magnetic resonance imaging evidence for cognitive reserve. Brain. 2010;133:362-74.

7. Sumowski JF, Wylie GR, Chiaravalloti N, DeLuca J. Intellectual enrichment lessens the effect of brain atrophy on learning and memory in multiple sclerosis. Neurology. 2010;74(24):1942-5.

8. Schwartz CE, Snook EM, Quaranto BR, Benedict RH, Vollmer T. Cognitive reserve and patient-reported outcomes. MS J. 2013;19(1):87-105.

9. Schwartz CE, Quaranto BR, Healy BC, Benedict RH, Vollmer T. Altruism and health outcomes in multiple sclerosis: The effect of cognitive reserve. J Positive Psychology. 2013;8(2):144-52.

10. Schwartz CE, Ayandeh A, Rodgers J, Duberstein P, Weinstock-Guttman B, Benedict $\mathrm{RH}$. A new perspective on proxy report: Investigating implicit processes of understanding through patient-proxy congruence. Quality of Life Research. 2015;24(11):2637-49.

11. Schwartz CE, Snook E, Quaranto B, Benedict RH, Rapkin BD, Vollmer T. Cognitive reserve and appraisal in multiple sclerosis. Multiple Sclerosis Related Disorders. 2013;2(1):36-44.

12. ECTRIMS. Time matters in multiple sclerosis-international consensus recommendations on diagnosis, management and access to treatment. Barcelona: ECTRIMS; 2015.

13. Sumowski JF, Chiaravalloti N, Wylie G, Deluca J. Cognitive reserve moderates the negative effect of brain atrophy on cognitive efficiency in multiple sclerosis. J Int Neuropsychol Soc. 2009;15(4):606-12.

14. Sumowski JF, Rocca MA, Leavitt VM, Riccitelli G, Comi G, Deluca J, et al. Brain reserve and cognitive reserve in multiple sclerosis: What you've got and how you use it. Neurology. 2013;80(24):2186-93.

15. Booth AJ, Rodgers JD, Schwartz CE, Quaranto BR, Weinstock-Guttman B, Zivadinov $\mathrm{R}$, et al. Active cognitive reserve influences the regional atrophy to cognition link in multiple sclerosis. J Int Neuropsychol Soc. 2013;19(10):1128-33.

16. IRTFIT. Software for the computation of the statistics involved in item response theory likelihood-ratio tests for differential item functioning [program]. UNC at Chapel Hill; 2001

17. Cappellani R, Bergsland N, Weinstock-Guttman B, Kennedy C, Carl E, Ramasamy $D$, et al. Subcortical deep gray matter pathology in patients with multiple sclerosis is associated with white matter lesion burden and atrophy but not with cortical atrophy: a diffusion tensor MRI study. AJNR Am J Neuroradiol. 2014:35(5):912-19.

18. Gabelic T, Ramasamy DP, Weinstock-Guttman B, Hagemeier J, Kennedy C, Melia $\mathrm{R}$, et al. Prevalence of radiologically isolated syndrome and white matter signal abnormalities in healthy relatives of patients with multiple sclerosis. AJNR Am J Neuroradiol. 2014;35(1):106-12.

19. O'Connor K, Weinstock-Guttman B, Carl E, Kilanowski C, Zivadinov R, Ramanathan M. Patterns of dietary and herbal supplement use by multiple sclerosis patients. J Neurol. 2012;259(4):637-44.

20. Zivadinov R, Marr K, Cutter G, Ramanathan M, Benedict RH, Kennedy C, et al. Prevalence, sensitivity, and specificity of chronic cerebrospinal venous insufficiency in MS. Neurology. 2011;77(2):138-144.

21. Perneczky R, Drzezga A, Boecker H, Ceballos-Baumann AO, Granert O, Förstl H, et al. Activities of daily living, cerebral glucose metabolism, and cognitive reserve in Lewy body and Parkinson's disease. Dement Geriatr Cogn Disord. 2008;26(5):475-81.

22. Zivadinov R, Heininen-Brown M, Schirda CV, Poloni GU, Magnano CR, Durfee J, et al. Abnormal subcortical deep-gray matter susceptibility- 
weighted imaging filtered phase measurements in patients with multiple sclerosis A case-control study. Neuroimage. 2012;59:331-39.

23. Gelineau-Morel R, Tomassini V, Jenkinson M, Johansen-Berg H, Matthews PM, Palace J. The effect of hypointense white matter lesions on automated gray matter segmentation in multiple sclerosis. Hum Brain Mapp. 2012;33: 2802-14.

24. Patenaude B, Smith S, Kennedy D, Jenkinson M. A Bayesian model of shape and appearance for subcortical brain segmentation. Neuroimage. 2011;56: 907-22.

25. Modica CM, Bergsland N, Dwyer MG, Ramasamy DP, Carl E, Zivadinov R, et al. Cognitive reserve moderates the impact of subcortical gray matter atrophy on neuropsychological status in multiple sclerosis. Multiple sclerosis. 2015;28: 1352458515579443.

26. Sormani MP, Kappos L, Cohen J, Barkhof F, Sprenger T, Meier DP, et al. Normalized brain volume predicts cognitive performance in MS: an analysis of a large cohort from fingolimod phase III studies (P7. 284). Neurology. 2015;84(14 (Supplement):P7-284.

27. Zar JH. Biostatistical Analysis. 2nd ed. Prentice Hall: Englewood Cliffs; 1984.

28. Stata 13 [program]. College Station. TX: StataCorp LP; 2013.

29. RStudio [program]. 0.98.1103 version. Boston: R Studio Inc; 2009-2014

30. van Praag H, Christie BR, Sejnowski TJ, Gage FH. Running enhances neurogenesis, learning, and long-term potentiation in mice. Proc Natl Acad Sci U S A. 1999; 96(23):13427-31.

31. Aguiar Jr AS, Castro AA, Moreira EL, Glaser V, Santos AR, Tasca Cl, et al. Short bouts of mild-intensity physical exercise improve spatial learning and memory in aging rats: involvement of hippocampal plasticity via AKT, CREB and BDNF signaling. Mech Ageing Dev. 2011;132(11-12):560-7.

32. Lovden M, Wenger E, Martensson J, Lindenberger U, Backman L. Structural brain plasticity in adult learning and development. Neurosci Biobehav Rev. 2013;37(9, Part B):2296-310.

33. Cotman CW, Berchtold NC. Exercise: a behavioral intervention to enhance brain health and plasticity. Trends Neurosci. 2002;25(6):295-301.

\section{Submit your next manuscript to BioMed Central and we will help you at every step:}

- We accept pre-submission inquiries

- Our selector tool helps you to find the most relevant journal

- We provide round the clock customer support

- Convenient online submission

- Thorough peer review

- Inclusion in PubMed and all major indexing services

- Maximum visibility for your research

Submit your manuscript at www.biomedcentral.com/submit

C Biomed Central 